\title{
Micromachining of passive planar micromixer on poly (methyl methacrylate) substrate with excimer laser ablation
}

\author{
Heng Qi · Yuhong Liu · Xuan Wang · Feng Shen · \\ Yong Yu · Tao Chen · Tiechuan Zuo
}

Received: 8 January 2008/Accepted: 6 June 2008/Published online: 28 June 2008

(c) Springer-Verlag 2008

\begin{abstract}
Excimer laser ablation technique was introduced into this work to fabricate a passive planar micromixer on the PMMA substrate. T-junction shaped and width-changed S-shaped microchannels were both designed in this micromixer to enhance mixing effect. The mixing experiment of distilled water and Rhodamine $\mathrm{B}$ with injection flow rate of 500 and $1,500 \mu \mathrm{m} / \mathrm{s}$ validates the mixing effectivity of this micromixer, and indicates the feasibility of excimer laser ablation in the microfabrication of $\mu$-TAS device.
\end{abstract}

\section{Introduction}

Microfluidic systems have been widely applied in biochemical, biological and chemical analysis for their potentials and advantages, such as small amounts of sample and reagent, less time consumption, lower cost and high throughput. Micromixer is a main component in the microfluidic system or $\mu$-TAS (micro total analysis system) because of the importance of the rapid and efficient mixing process in many unit operations, including heat exchange or chemical reaction. Different mixing principles and micromixer designs have been reported in order to enhance fluid mixing within the microchannel. Micromixers can be

H. Qi $(\bowtie) \cdot$ T. Chen · T. Zuo

Institute of Laser Engineering, Beijing University

of Technology, 100124 Beijing, China

e-mail: qiheng2000@emails.bjut.edu.cn

Y. Liu $\cdot$ X. Wang $\cdot$ F. Shen $\cdot$ Y. Yu

National Microgravity Laboratory, Institute of Mechanics,

Chinese Academy of Science, 100080 Beijing, China mainly categorized as active micromixers and passive micromixers. Active micromixers generally require external power source for mixing enhancement, such as mechanical (Evans et al. 1997), acoustic/ultrasonic (Yang et al. 2001; Liu et al. 2003), electrokinetic (Oddy et al. 2001), magnetic (Bao et al. 2001; Lu et al. 2002) or thermal source (Tsai and Lin 2002). Active micromixers have higher mixing efficiency; the mixing time and microchannel length required for uniform mixing are less than those for passive micromixers. However, the requirement of external power makes them difficult to be integrated with other microfluidic devices. Furthermore, the relatively high power consumption and cost make active mixers less attractive for disposable applications. Contrarily, passive micromixers are simple to operate because the mixing process occurs along with the structure change of microchannel, making them attractive and suitable for integration with other devices. The passive micromixers mainly have two types of mixing mechanisms namely chaotic advection (Liu et al. 2000; Kim et al. 2004; Bhagat et al. 2007) and lamination (Wu and Nguyen 2005; Ducrée et al. 2006). In chaotic advection micromixers, 3D channel structures and planar designs were used to enhance fluid mixing. The fabrication of 3D structure and multiple-layer mixers is much more complicated, but the planar micromixer is easily integrated with the microfluidic system. In the geometrical designs of planar micromixers, the methods of adding the obstructions into the microchannel were often used. The other type of passive micromixers is the lamination micromixers based on molecular diffusion. Uniform mixing can be achieved by lamination at lower Reynolds number.

The most common used substrate materials in fabrication of micromixer include silicon, quartz, glass and polymers. As one primary kind of thermoplastic polymer, 
poly (methyl methacrylate) (PMMA) has been widely used for the fabrication of microfluidic devices. In this research, T-junction shaped and width-changed S-shaped microchannels were designed in the micromixer configuration to enhance mixing effect. Excimer laser direct writing ablation technique was introduced into this work to fabricate the micromixer on PMMA substrate. The fabrication time and cost could be decreased observably, especially in the step of lab research because of the flexibility of laser fabrication and low price of polymer. The mixing effect of micromixer was viewed by simple mixing experiment between distilled water and Rhodamine B. The main purpose of this work was to validate the mixing effectivity of this micromixer configuration, and indicate the feasibility of excimer laser ablation in the microfabrication of $\mu$-TAS device.

\section{Experimental}

\subsection{Micromixer configuration and fabrication}

The configuration of the passive planar micromixer was designed, as shown in Fig. 1, with exterior chip size of $70 \mathrm{~mm} \times 65 \mathrm{~mm}$.

In this configuration, four silicone capillary tubes were in advance connected between the holes H3 and H9, H4 and $\mathrm{H} 8, \mathrm{H} 5$ and $\mathrm{H} 7, \mathrm{H} 6$ and $\mathrm{H} 10$, respectively. Rhodamine B solution and deionized water were injected into the microchannel from Inlet 1 and Inlet 2, respectively. Rhodamine B solution was separated as two streams in the T-junction (T1) of Species A and respectively flowed into the holes $\mathrm{H} 3$ and $\mathrm{H} 4$, and then they were introduced into the holes $\mathrm{H} 8$ and $\mathrm{H} 9$ through the capillary tubes. Similarly, the deionized water was also introduced into the holes H7 and H10. Two streams introduced from the holes $\mathrm{H} 7$ and $\mathrm{H} 9$ were concentrated into one stream at T3 of Species $\mathrm{C}$ and farther concentrated into a brand new stream with other stream, which was concentrated at $\mathrm{T} 4$ between the Rhodamine B solution and the deionized water introduced from the holes $\mathrm{H} 8$ and H10, respectively. The new concentrated stream at T5 flowed along with the microchannel M1 and orderly passed through M2, M3, M4 and M5. The dimension variety of cross section between these microchannels resulted in the change of flow rate. Moreover, the existence of M3 and the position difference between M2 and M3 caused the flow turbulence of stream. After that, the stream was decelerated and flowed to Outlet along with microchannel M5. Finally, the stream was collected with a micro container.

The depths of all microchannels were all $200 \mu \mathrm{m}$. The widths of microchannels M1, M3 and M5 were all $1 \mathrm{~mm}$. The widths of M2 and M3 were $250 \mu \mathrm{m}$. The widths of other microchannels were all $500 \mu \mathrm{m}$.

The micromixer was fabricated on PMMA substrate. PMMA material with thickness of $1 \mathrm{~mm}$ was purchased from China An' hui Shuguang Chemical Group, and precut with low power CO2 laser machine (F1-30 W, HM Laser Machinery Co., LTD, China) to definite-sized substrates $(70 \mathrm{~mm} \times 65 \mathrm{~mm})$. Before any type of experimental procedure was carried out, PMMA substrates were sonicated in deionized water for $10 \mathrm{~min}$, and subsequently dried under pressurized air.
Fig. 1 Schematic diagram of the micromixer on the PMMA substrate

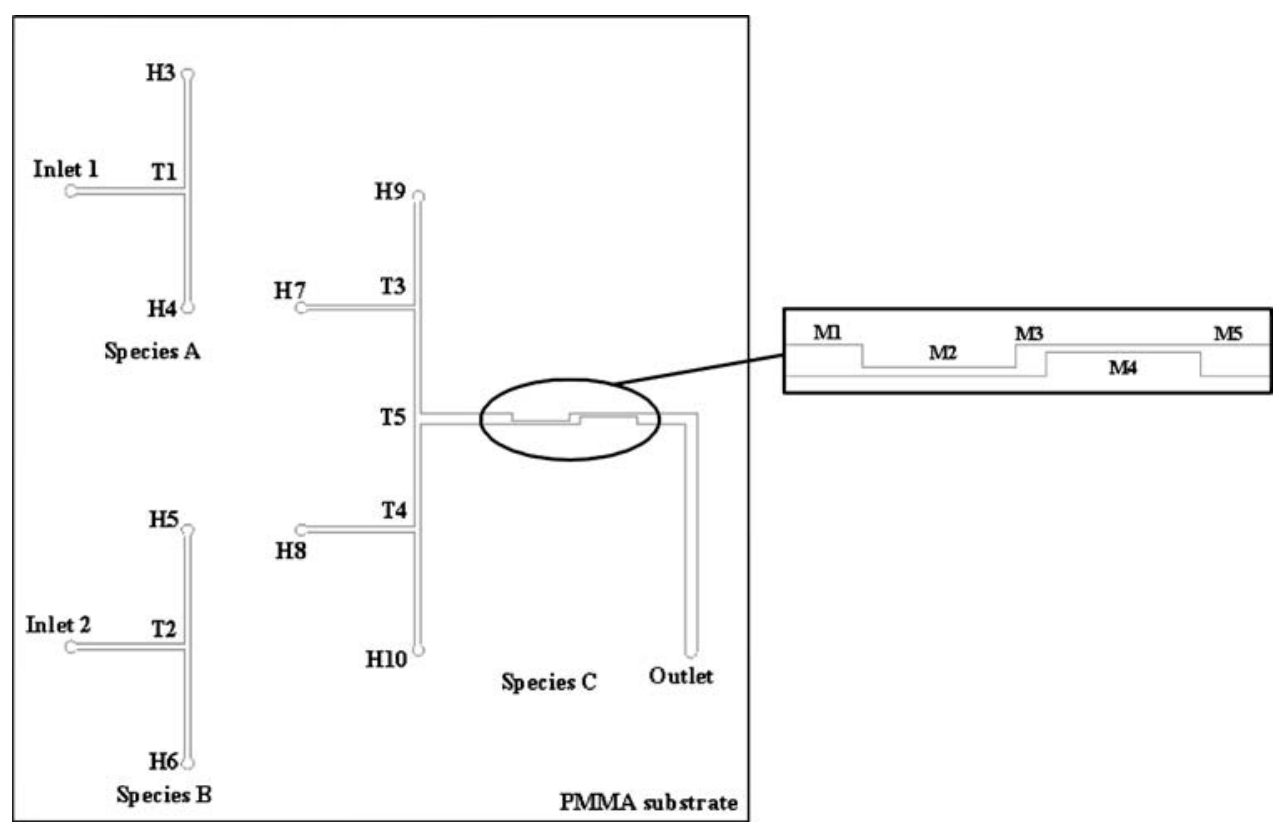


Fig. 2 Three-dimensional simulation profiles of microchannels in T1 (a) and M3 (b)
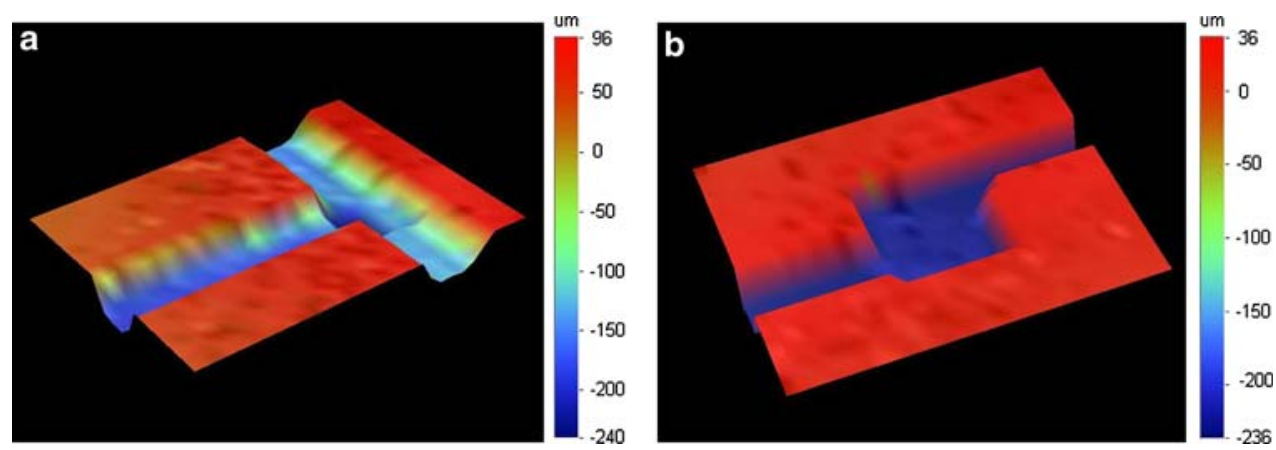

In this work, a commercially excimer laser machine (LPX305iF, Lambda Physik, Germany) was used to fabricate microchannels on PMMA substrate. Its emission wavelength is $248 \mathrm{~nm}$, the maximum energy is $1,200 \mathrm{~mJ}$ per pulse and the maximum repetition frequency is $50 \mathrm{~Hz}$ with pulse duration of $30 \mathrm{~ns}$. The translational speed of working platform could be adjusted from 1.5 to $60 \mathrm{~mm} /$ min. The reduction ratio of objective lens is 10:1. In this study, three masks, which have sizes of $10 \mathrm{~mm} \times 10 \mathrm{~mm}$, $5 \mathrm{~mm} \times 5 \mathrm{~mm}$ and $2.5 \mathrm{~mm} \times 2.5 \mathrm{~mm}$, were used to gain different sizes of laser beam projected on the surface of PMMA substrate to fabricate different microchannels. The fluence of excimer laser and the repetition pulse frequency were set as $7.02 \mathrm{~J} / \mathrm{cm}^{2}$ and $20 \mathrm{~Hz}$; and different translational speeds of working platform were adjusted from $2 \mathrm{~mm} / \mathrm{min}$ to $14 \mathrm{~mm} / \mathrm{min}$ based on the different sizes of masks. During fabrication, the ablation area was continuously blown with compressed air of $0.2 \mathrm{MPa}$ to blow away the generated impurity in microchannel. After ablation of microchannel, the PMMA substrate was irradiated by excimer laser with lower fluence of $0.18 \mathrm{~J} / \mathrm{cm}^{2}$ for 30 times to make polishing processing for further improving the surface roughness of microchannels. The profile of the microchannel on the PMMA substrate was viewed and measured with the non-contact 3D optical profiling system (Wyko NT1100, Veeco Instrument Inc., USA).

The PMMA substrate ablated with microchannels was bonded together with other plain PMMA cover substrate to form a closed structure using hot-press bonding technique with the home-built spring press machine, which was placed in a constant temperature electric heater and heated for $75 \mathrm{~min}$ at $105^{\circ} \mathrm{C}$. Finally, the bonded micromixer chip was naturally cooled down to room temperature inside the spring press machine.

\subsection{Mixing experiment}

The mixing effect of the passive planar micromixer was charactered using Rhodamine B solution and deionized water. A home-built simple air-operated sampling pump was used to deliver Rhodamine B solution and deionized water to
Inlet 1 and Inlet 2, respectively. The mixing result was viewed with an inverted confocal microscope. The injection flow rates used in this research were 500 and $1,500 \mu \mathrm{m} / \mathrm{s}$.

\section{Results and discussion}

The microstructure of microchannel was measured with 3D optical profiling system and analyzed and simulated using WYKO Vision32 software (WYKO Corporation, USA). The 3D simulation profiles of microchannels at T-junction $\mathrm{T} 1$ and S-shaped microchannel M3 were shown in Fig. 2a and $b$, respectively. The surface roughness $\mathrm{Ra}$ of microchannel was $2.2 \mu \mathrm{m}$.

The mixing effect was viewed with inverted confocal microscope. Figure 3 shows the mixing effect in microchannel M1 (a) and M5 (b), respectively, with different flow rates. Figure $3 \mathrm{a} 1$ shows the initial mixing effect at M1 with flow rate of $500 \mu \mathrm{m} / \mathrm{s}$. Because of the pre-mixing effects at T3 and T4, the stream in M1 consists of four streams, which come from the holes $\mathrm{H} 9, \mathrm{H} 7, \mathrm{H} 8$ and $\mathrm{H} 10$, respectively. When the stream passed through the widthchanged S-shaped microchannels (M2, M3 and M4), it was further mixed by the turbulence effect of the designed microstructure and became uniform in distribution of two samples, when flowed to M5, as shown in Fig. 3b1. When the flow rate increased to $1,500 \mu \mathrm{m} / \mathrm{s}$, the stream in M1 had four more distinct streams because of the weakness of the diffusion effect in the straight microchannels, as shown in Fig. 3a2. However, Fig. $3 \mathrm{~b} 2$ shows that same final mixing effect could be received in M5 after the stream passed through the width-changed S-shaped microchannels. From Fig. 3, we can conclude that this passive planar micromixer configuration has obvious mixing effect under different flow rates in microchannels.

\section{Conclusion}

In this study, excimer laser direct writing ablation technique was introduced to fabricate passive planar micromixer on 
Fig. 3 Mixing effects in microchannels M1 (a) and M5 (b) with flow rate of 500 and $1,500 \mu \mathrm{m} / \mathrm{s}$
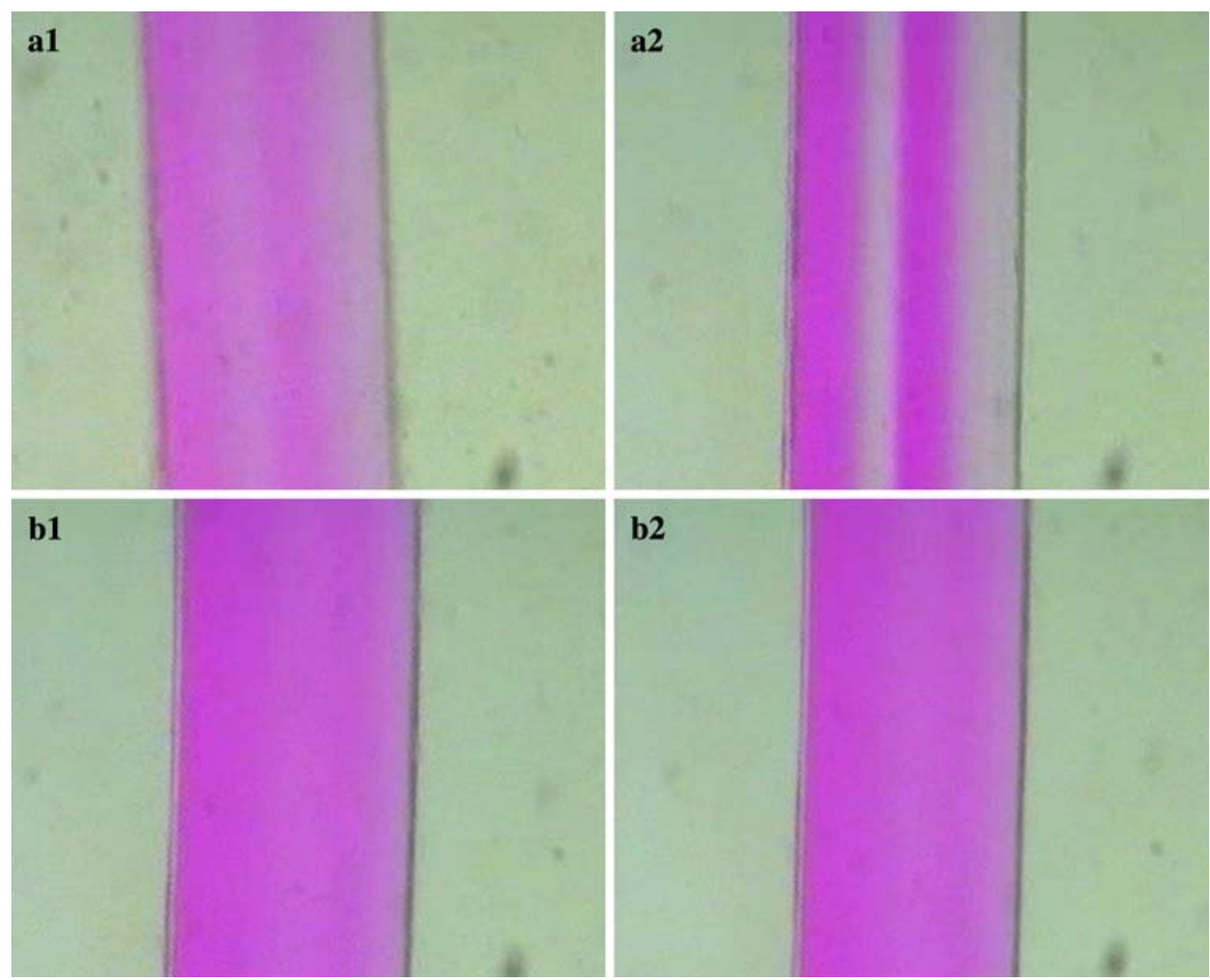

the PMMA substrate. T-junction shaped and width-changed $\mathrm{S}$-shaped microchannels were designed in this micromixer to enhance mixing effect. The mixing experiment of Rhodamine $\mathrm{B}$ and deionized water with different flow rates well validates the mixing effectivity of this micromixer configuration, and indicates the feasibility of excimer laser ablation used in microfabrication of $\mu$-TAS device.

Acknowledgments This work was supported by National Natural Science Foundation of China (50335050) and Beijing Natural Science Foundation (3031001) and Funding Project for Academic Human Resources Development in Institutions of Higher Learning under the Jurisdiction of Beijing Municipality.

\section{References}

Bao HH, Zhong J, Yi M (2001) A minute magneto hydro dynamic (MHD) mixer. Sens Actuators B 79:207-215. doi:10.1016/ S0925-4005(01)00851-6

Bhagat AAS, Peterson ETK, Papautsky I (2007) A passive planar micromixer with obstructions for mixing at low Reynolds numbers. J Micromech Microeng 17:1017-1024. doi:10.1088/ 0960-1317/17/5/023

Ducrée J, Brenner T, Haeberle S, Glatzel T, Zengerle R (2006) Multilamination of flows in planar networks of rotating microchannels. Microfluid Nanofluid 2:78-84. doi:10.1007/s10404-0050056-5
Evans J, Liepmann D, Pisano AP (1997) Planar laminar mixer. Proceedings of MEMS'97. IEEE, Nagoya, pp 96-101

Kim DS, Lee SW, Kwon TH, Lee SS (2004) A barrier embedded chaotic micromixer. J Micromech Microeng 14:798-805. doi: 10.1088/0960-1317/14/6/006

Liu RH, Stremler MA, Sharp KV, Olson MG, Santiago JG, Adrian RJ et al (2000) Passive mixing in a three-dimensional serpentine microchannel. J Microelectromech Syst 9:190-197. doi: $10.1109 / 84.846699$

Lu LH, Ryu KS, Liu C (2002) A magnetic microstirrer and array for microfluidic mixing. J Microelectromech Syst 11:462-469. doi: 10.1109/JMEMS.2002.802899

Liu RH, Lenigk R, Druyor-Sanchez RL, Yang JN, Grodzinski P (2003) Hybridization enhancement using cavitation microstreaming. Anal Chem 75:1911-1917. doi:10.1021/ac026267t

Oddy MH, Santiago JG, Mikkelsen JC (2001) Electrokinetic instability micromixing. Anal Chem 73:5822-5832. doi:10.1021/ ac0155411

Tsai JH, Lin LW (2002) Active microfluidic mixer and gas bubble filter driven by thermal bubble micropump. Sens Actuators A 97-98:665-671. doi:10.1016/S0924-4247(02)00031-6

Wu ZG, Nguyen NT (2005) Convective-diffusive transport in parallel lamination micromixers. Microfluid Nanofluid 1:208-217. doi: 10.1007/s10404-004-0011-x

Yang Z, Goto H, Matsumoto M, Maeda R (2001) Ultrasonic micromixer for microfluidic systems. Sens Actuators A 93:266-272. doi:10.1016/S0924-4247(01)00654-9 